\title{
SURVIVAL OF STAPHYLOCOCCUS AUREUS IN INTRAPERITONEAL ABSCESSES
}

\author{
E. S. DYE* AND F. A. KAPRAL \\ Department of Medical Microbiology, The Ohio State University, Columbus, Ohio \\ 43210, USA
}

SUMmary. An examination of 10 strains of Staphylococcus aureus for survival within abscesses developing in the peritoneal cavity of mice revealed three distinct patterns of survival. Although non-haemolytic mutants were destroyed more rapidly than were their parent strains, this difference could not be attributed to any particular haemolysin. In abscesses generated with mixtures of non-haemolytic variants and their parent strains, the former were preferentially eliminated; this suggests that the non-haemolytic variants were inherently more sensitive to the conditions within these lesions. Subsequent studies confirmed that abscess homogenates were cidal for staphylococci and that this activity resided in the insoluble fraction of the homogenates. Staphylococci added to abscess homogenates were killed, but only after a lag. This lag could be shortened or eliminated by incubating homogenates before adding the test organism. After development of a suitable assay, it was found that the cidal activity in abscess homogenates could be increased 3-20-fold by pre-incubation. Staphylococcal strains differed in their relative sensitivities to the cidal material; those strains rapidly destroyed within abscesses were the most sensitive and strains capable of better survival were more resistant. The results support the concept that the cidal material is responsible for destruction of staphylococci within such lesions.

\section{INTRODUCTION}

Although the abscess is the most common form of the staphylococcal lesion, little is known about factors governing the behaviour of Staphylococcus aureus in such sites. This is due, in part, to a paucity of suitable experimental models for studying these problems. During an investigation of the fate of staphylococci in the peritoneal cavity of mice, it was realised that this route of infection could be used to generate abscesses from which material is recoverable with ease and free from extraneous host tissue (Kapral, Godwin and Dye, 1980). Although large numbers of staphylococci are required for establishing these abscesses and early multiplication of the inoculum has not been observed, 
this model has proved useful for the study of the complex host-parasite interactions that influence the organism's survival within these lesions.

The intraperitoneal inoculation of $2 \times 10^{8}$ or more non-capsulate $S$. aureus into mice results in prompt clumping of the organisms by the interaction of fibrinogen in the peritoneal fluid with clumping factor $(C F)$ on the surface of the cocci (Kapral, 1966). During the next $3 \mathrm{~h}$, neutrophils summoned by the ensuing inflammatory response adhere to the clumps and completely enclose them with a thick layer of leukocytes. During the next 48-72 h the structures are successively covered by fibrin and connective tissue, giving rise to lesions histologically resembling typical staphylococcal abscesses (Kapral et al., 1980).

The clumping reaction is an effective mechanism for protecting the inoculum from phagocytes. Although the availability of CF on the surface is an important determinant, the number of staphylococci injected is also a critical factor for the development of these abscesses. Strains lacking CF are eliminated by phagocytosis even when large doses are introduced into the peritoneal cavity (Kapral, 1966). However, even with strains possessing CF, the inoculation of less than $2 \times 10^{8}$ cocci results in inefficient clumping and this leaves the organisms subject to phagocytosis by peritoneal macrophages.

Even though the clumped organisms become surrounded by leukocytes, phagocytosis is minimal and almost the entire inoculum is initially present in the few resultant abscesses (Kapral, 1966; Kapral et al., 1980). While staphylococci are subsequently killed, the process is not attributable to phagocytosis or the release of microbicidal lysosomal substances. Previous studies suggest that the appearance of a staphylocidal factor, which can be detected in abscess homogenates, accounts for destruction of organisms within such lesions (Dye and Kapral, 1980). This material, associated with the insoluble fraction of abscess homogenates, is thermostable and appears to be a lipid.

Because prior findings suggested a possible relationship between a strain's survival within abscesses and its ability to produce $\alpha$ toxin (Kapral et al., 1980), further studies were undertaken to evaluate this relationship. Accordingly, strains possessing different combinations of toxins were examined for their ability to survive within abscesses. It was found that $\alpha$-toxin production alone did not determine survival in these lesions, but the ability to survive within abscesses was correlated with a strain's resistance to the bactericidal substance in abscess homogenates.

\section{MATERIALS AND METHODS}

\section{Animals}

Sprague-Dawley White Swiss female mice weighing approximately $25-30 \mathrm{~g}$ were used throughout these studies.

\section{Staphylococcal strains}

S. aureus strains 18Z, P78, PG114, TG, and 689 have been previously described (Kapral and Miller, 1971; Kapral and Shayegani, 1959; Melish and Glasgow, 1970). Strains 18Z and P78 produced both the $\alpha$ and $\delta$ toxins, whereas strain TG elaborated $\alpha, \beta$ and $\delta$ toxins. Strain 689 produced only $\alpha$ toxin and strain PG114 only the $\delta$ toxin. S. aureus strain 303, producing only the $\beta$ toxin, was obtained from the departmental stock culture collection and is of obscure origin. All strains were capable of producing the soluble coagulase and clumping factor. The $\alpha$-toxinnegative variants, 18Z-G, 18Z-H, P78-22, and the $\delta$-toxin-negative variant PG114-1, were derived from their respective parents after ultraviolet irradiation.

The variants $18 \mathrm{Z} \mathrm{Sm} \mathrm{r}^{\mathrm{r}}, \mathrm{P} 78 \mathrm{Sm}^{\mathrm{r}}$ and $\mathrm{P} 78-22 \mathrm{Sp}^{\mathrm{r}}$, resistant to either streptomycin or spectino- 
mycin, were derived from their respective parents by plating on trypticase soy agar (TSA) containing either streptomycin $60 \mu \mathrm{g} / \mathrm{ml}$ or spectinomycin $600 \mu \mathrm{g} / \mathrm{ml}$.

\section{Suspensions for animal inoculation}

Staphylococcal strains were grown in trypticase soy broth (TSB) under a constant airflow while agitated on an orbital shaker. After incubation for $24 \mathrm{~h}$ at $37^{\circ} \mathrm{C}$, cultures were centrifuged in the cold, and the cells washed three times in normal saline with $0.1 \%$ trypticase and $0.1 \%$ skim milk. The staphylococci were resuspended in the same diluent to give a final concentration of approximately $8 \times 10$ cells $/ \mathrm{ml}$, and the resultant suspensions sealed in ampoules and stored at $-70^{\circ} \mathrm{C}$. Plate counts were done on suspensions at the time of preparation and at the time of use.

\section{Generation of intraperitoneal abscesses and enumeration of viable cocci}

Groups of 40-50 mice were inoculated intraperitoneally with 1-3 $\times 10^{9}$ staphylococci in 0.25 $\mathrm{ml}$ of diluent. This dose permitted adequate intraperitoneal clumping of the organisms while maximising survival of the test animals. At various intervals after inoculation, 4-8 mice were killed and the intraperitoneal abscesses were aseptically removed. Initial samples were obtained $4 \mathrm{~h}$ after infection when layering of leukocytes about the staphylococcal clumps was sufficient to permit recovery of the inoculum from the peritoneal cavity. Additional samples were collected over a 4-5-week period. Abscesses from a single animal were pooled and homogenised in $2 \mathrm{ml}$ of sterile saline with a glass tissue grinder fitted with a motor-driven teflon pestle. After initial homogenisation, each homogenate was diluted with $3 \mathrm{ml}$ of trypsin solution (Difco Trypsin $1: 25010 \mathrm{mg} / \mathrm{ml}$ diluted 1 in 250 in $0.1 \mathrm{M}$ phosphate buffer, $p \mathrm{H} 7.8$ ) and rehomogenised. The abscess homogenates were allowed to stand for $30 \mathrm{~min}$ at room temperature to assist dissociation of clumped staphylococci. The suspensions were again homogenised, serially diluted, and plated on TSA.

In the infection studies with mixtures of two strains of staphylococci, mutants of the respective strains were selected such that one was resistant to streptomycin and the other resistant to spectinomycin. The population of each strain was subsequently counted by plating portions of abscess homogenates on TSA containing either streptomycin $(60 \mu \mathrm{g} / \mathrm{ml})$, or spectinomycin $(600 \mu \mathrm{g} / \mathrm{ml})$. Samples were also plated on medium without antibiotics to verify the total number of organisms present.

\section{Preparation of abscess homogenates for evaluation of bactericidal activity}

Abscesses were generated by the intraperitoneal inoculation of $10^{9}$ organisms of $S$. aureus strain 18Z. After the desired interval, abscesses from 20 mice were harvested, pooled, and homogenised in $20 \mathrm{ml}$ of saline. The resultant homogenate was sealed in ampoules and stored at $-70^{\circ} \mathrm{C}$ until needed.

After initial findings had revealed that bactericidal activity was associated with the insoluble fraction of abscess homogenates, homogenates were processed further by centrifugation at $40000 \mathrm{~g}$ for $30 \mathrm{~min}$ in the cold. The supernatant fraction was discarded and the sediment together with floating film was washed three times with saline, resuspended in the same diluent at the original volume, and stored at $-70^{\circ} \mathrm{C}$. This type of preparation is referred to as pelleted abscess homogenate (PAH).

Assay of bactericidal activity. After studies (Dye and Kapral, 1980) had indicated that the bactericidal activity of abscess homogenates was heat stable, heating at $100^{\circ} \mathrm{C}$ for $30 \mathrm{~min}$ was used to eliminate residual viable staphylococci from homogenates before the addition of the indicator organism used in the assay. S. aureus strain $18 \mathrm{Z}-\mathrm{G}$, which served as the indicator organism, was prepared by diluting a frozen washed suspension in diluent consisting of saline with $8 \%$ TSB to give a final concentration of $10^{3} \mathrm{cocci} / \mathrm{ml}$.

Serial twofold dilutions of boiled PAH were made in either saline or $2 \mathrm{M} \mathrm{NaCl}$ containing $2 \mathrm{~mm}$ EDTA. To $0.5-\mathrm{ml}$ amounts of each PAH dilution was added $0.5 \mathrm{ml}$ of the indicator 
organism suspension. After incubation for $1 \mathrm{~h}$ at $37^{\circ} \mathrm{C}$, the surviving cocci were enumerated by plating $0.5-\mathrm{ml}$ portions from each dilution in TSA. A minimum of three tubes containing diluent and the indicator organism suspension served as controls. Each assay was done in triplicate. A cidal unit (LD50) was defined as the quantity of PAH that destroyed $50 \%$ of the staphylococci during the $1-\mathrm{h}$ incubation period, and the concentration of cidal activity was expressed as LD $50 / \mathrm{ml}$. The $50 \%$ endpoints were determined by the method of probits (Finney, 1971). Statistical analysis of LD50 values obtained from multiple assays on the same PAH suspension indicated a $95 \%$ confidence interval for the mean LD50 of $\pm 20 \%$.

\section{RESULTS}

\section{Survival of staphylococci within abscesses}

After study of the survival within abscesses of 10 strains of $S$. aureus, three general patterns of survival were evident (fig. 1). S. aureus strain TG persisted for up to 5 weeks without undergoing any significant population change. The persistence of $S$. aureus strain PG114 and $18 \mathrm{Z}$ was characterised during the first 7-14 days by a population decrease, followed by a population increase to approximately original levels. Thereafter the number of cocci again decreased. For the remaining strains the populations declined promptly and continuously, albeit at different rates. Whilst no particular combination of haemolysins proved uniformly beneficial for the survival of a strain within abscesses, the $\alpha$-toxin-negative variants 18Z-G, 18Z-H, P78-22 and the $\delta$-negative PG114-1, were more readily destroyed than were their toxigenic parents.

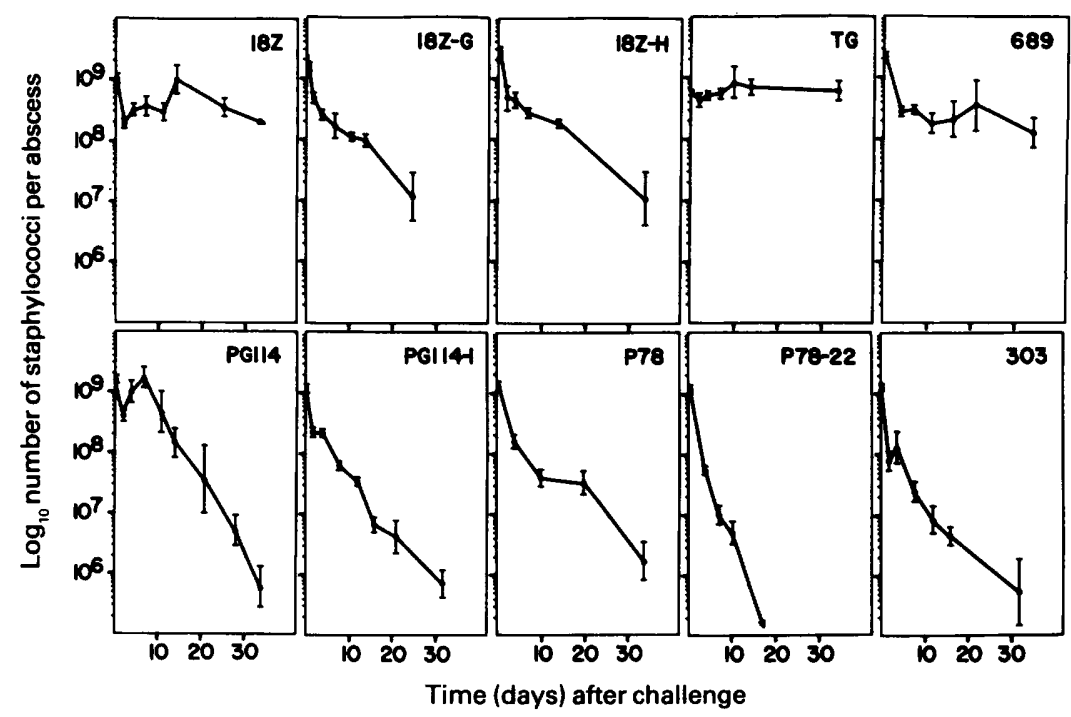

Fig. 1.-Survival of various strains of $S$. aureus in abscesses. Each point represents the geometric mean $( \pm \mathrm{SE}$ ) of results from four animals. The first point gives the number of organisms recovered from an abscess at first sampling at $4 \mathrm{~h}$. 
Mixed infection with haemolytic and non-haemolytic strains

To determine whether haemolysin production within abscesses might alter some host defence mechanism and thus influence the survival of staphylococci within abscesses, mixtures of parent strains and their derived nonhaemolytic variants were inoculated intraperitoneally into mice.

The results of two such studies are presented in figs. 2 and 3. In each case the survival of the two strains when present together was essentially the same as when they were present alone. This suggested that the preferential destruction of non-haemolytic strains might be the result of their greater sensitivity to some host material in the abscess.

\section{Development of bactericidal activity in abscess homogenates}

Because previous studies had indicated that destruction of staphylococci added to abscess homogenates occurred only after a lag period (Kapral et al., 1980), we elected to examine this phenomenon in greater detail. Addition of the 18Z-G strain to abscess homogenates, or the pelleted fraction thereof, revealed the existence of a 4-8-hour lag before the onset of cidal activity. This lag could be shortened by incubating the pelleted homogenate (PAH) at $37^{\circ} \mathrm{C}$ for increasing periods before the addition of the test organism (fig. 4). A 24-hour pre-incubation ("activation") of PAH resulted in a preparation that was capable of reducing the initial population to less than $1 \%$ in $1 \mathrm{~h}$.

To investigate the activation process further, an assay was designed to measure the cidal activity in PAH. PAH was incubated in saline at $37^{\circ} \mathrm{C}$ and

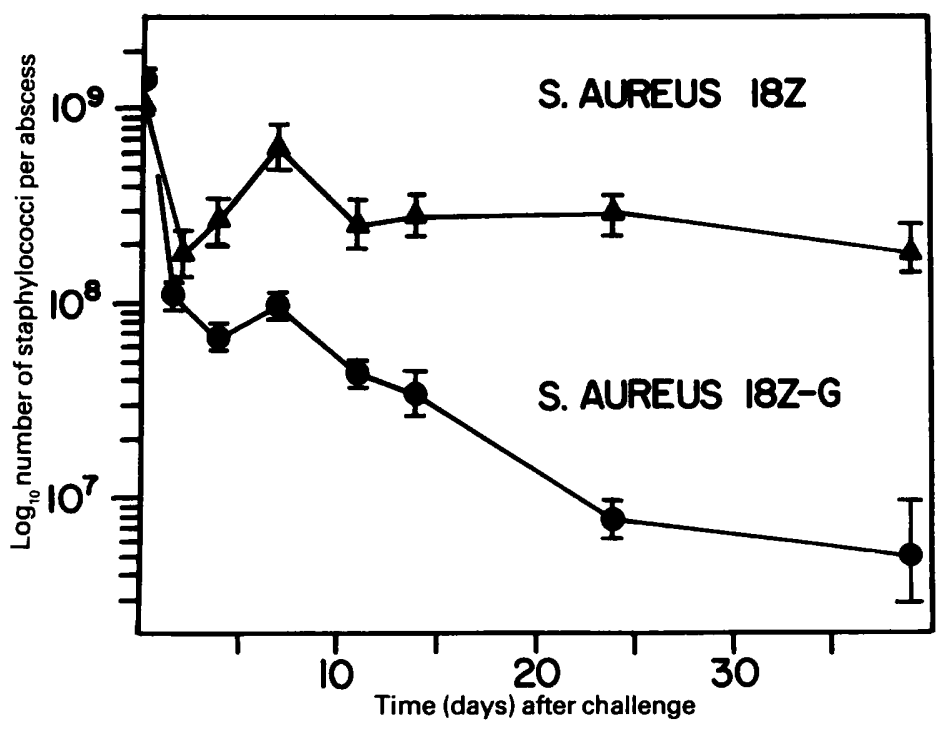

FIG. 2.-Survival of $S$. aureus strains $18 Z$ (streptomycin resistant) and 18Z-G (spectinomycin resistant) when combined in abscesses. Each point represents the geometric mean $( \pm$ S.E. $)$ of results from 5-10 animals. 


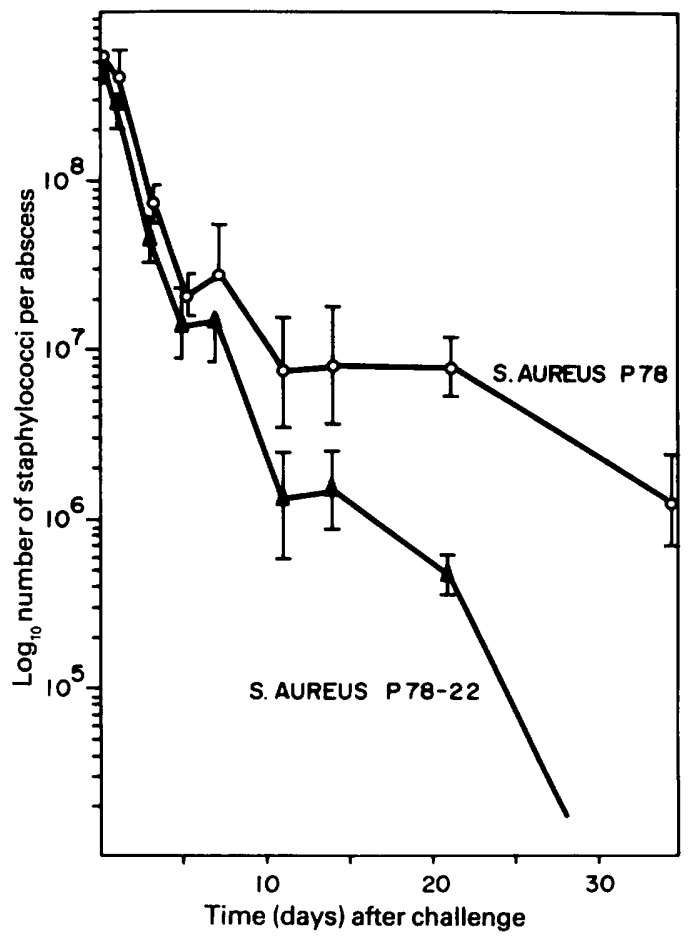

FIG. 3.-Survival of $S$. aureus strains P78 (streptomycin resistant) and P78-22 (spectinomycin resistant) when combined in abscesses. Each point represents the geometric mean $( \pm S$.E.) of results from four animals.

portions were periodically removed to measure changes in cidal activity during activation. In some studies, samples were centrifuged at $40000 \mathrm{~g}$ and the supernate and sediment were assayed separately. The results indicated that maximum levels of cidal activity were attained after an 8-24-h period of incubation and the activity remained unchanged thereafter (table I). The supernate possessed little or no cidal activity; this suggests that the cidal material was not readily solubilised during the activation process. Activation of different PAH preparations revealed that cidal activity increased 3-20-fold over that initially present. In future studies $\mathrm{PAH}$ incubated for $24 \mathrm{~h}$ at $37^{\circ} \mathrm{C}$ was called "activated pelleted abscess homogenate" (AcPAH).

\section{Relative susceptiblity of staphylococcal strains to AcPAH}

To determine whether there is any relationship between a strain's sensitivity to the cidal substance and its ability to survive in abscesses, the strains previously studied in abscesses were evaluated for their sensitivity to AcPAH. By substituting these strains for the indicator organism and assaying the same AcPAH preparation, a series of endpoints was obtained that reflected the relative sensitivity of these strains to the cidal substance (table II). The greater the sensitivity of the strain used, the greater was the LD50. Sensitivity to 


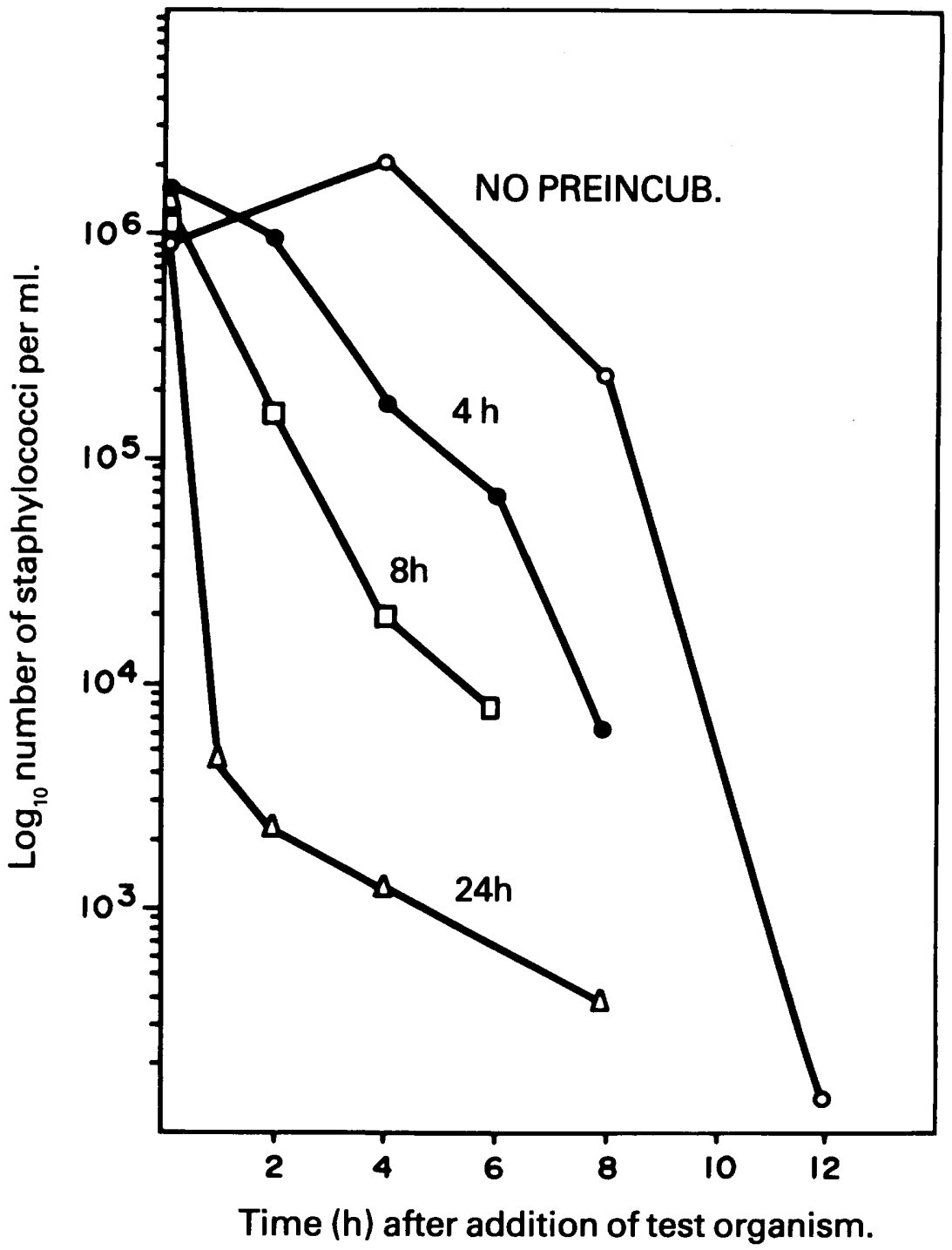

Fig. 4.-Effect of pre-incubating pelleted abscess homogenate on subsequent staphylococcal destruction. The homogenate from 7-day-old abscesses was diluted 1 in 5 in saline and incubated at $37^{\circ} \mathrm{C}$ for the periods indicated before addition of $S$. aureus strain 18Z-G (spectinomycin resistant) at a concentration of $10^{6} / \mathrm{ml}$. The test organisms were enumerated by plating samples on trypticase soy agar containing spectinomycin. S. aureus strain $18 \mathrm{Z}$, which was used to generate abscesses and which was still present in the homogenates, was sensitive to spectinomycin and did not interfere with counts of the test strain.

AcPAH varied markedly among the strains tested. Moreover, the relative resistance of a strain to AcPAH correlated with its ability to survive within abscesses. Strains that were immediately destroyed were the most sensitive, whilst $S$. aureus strain TG, characterised by prolonged persistence within abscesses, was the most resistant. S. aureus strains PG114 and 18Z, whose survival pattern in abscesses was characterised by a population rebound and a 
TABLE I

Activation of pelleted abscess homogenate

\begin{tabular}{|c|c|c|c|}
\hline \multirow{3}{*}{$\begin{array}{c}\text { Sampling } \\
\text { time } \\
\text { (h) }\end{array}$} & \multicolumn{3}{|c|}{$\mathrm{LD} 50 / \mathrm{ml}^{*}$ of } \\
\hline & \multicolumn{2}{|c|}{ pellet } & \multirow{2}{*}{$\begin{array}{l}\text { supernate } \\
\text { prep. } 2\end{array}$} \\
\hline & prep. 1 & prep. 2 & \\
\hline 0 & 150 & 240 & $<10$ \\
\hline 4 & 580 & & \\
\hline 8 & 670 & 800 & $<10$ \\
\hline 12 & 640 & & in \\
\hline 24 & 620 & 1000 & $<10$ \\
\hline $\begin{array}{l}48 \\
72\end{array}$ & $\cdots$ & $\begin{array}{l}870 \\
960\end{array}$ & $<10$ \\
\hline 96 & $\begin{array}{l}\cdots \\
\cdots\end{array}$ & 930 & 50 \\
\hline
\end{tabular}

* Assayed in saline; the indicator organism was $S$. aureus strain $18 \mathrm{Z}-\mathrm{G} ; \ldots=$ not done.

\section{TABLE II}

Comparison of relative sensitivities to activated pelleted abscess homogenate with rapidity of destruction of $S$. aureus strains in abscesses

\begin{tabular}{|c|c|c|}
\hline $\begin{array}{l}S . \text { aureus } \\
\text { strain }\end{array}$ & $\begin{array}{l}\text { Relative sensitivity } \\
\text { (LD50/ml)* }\end{array}$ & $\begin{array}{l}\text { Rapidity of } \\
\text { destruction in } \\
\text { abscesses }\end{array}$ \\
\hline $\begin{array}{l}303 \\
689 \\
\text { P78-22 } \\
\text { PG114-1 } \\
18 Z-G \\
\text { P78 } \\
18 Z-H \\
\text { PG114 } \\
18 Z \\
\text { TG }\end{array}$ & $\begin{array}{r}8000 \\
3200 \\
2700 \\
2300 \\
2200 \\
1600 \\
530 \\
350 \\
120 \\
40\end{array}$ & $\begin{array}{l}\text { Immediate } \\
\text { Immediate } \\
\text { Immediate } \\
\text { Immediate } \\
\text { Immediate } \\
\text { Immediate } \\
\text { Immediate } \\
\text { Delayed } \\
\text { Delayed } \\
\text { None in } 35 \text { days }\end{array}$ \\
\hline
\end{tabular}

delayed onset of final destruction, possessed intermediate sensitivity to the cidal substance.

\section{Discussion}

Earlier studies with three different strains of $S$. aureus suggested a possible relationship between a strain's ability to produce $\alpha$ toxin and its ability to survive within peritoneal abscesses (Kapral et al., 1980). In the present study, six $S$. aureus strains, differing with respect to haemolysins produced, and four derived mutants were examined for their ability to persist within abscesses. These studies failed to reveal a haemolysin that might be associated with long-term survival in abscesses. 
Three types of survival patterns were noted. With most strains destruction within abscesses began promptly and continued, although at different rates, for the period of observation.

The survival pattern of $S$. aureus strains $18 \mathrm{Z}$ and PG114 was characterised by an initial population decrease followed by an increase to near original levels. Thereafter the populations again declined but at different rates for the two strains. This "rebound" phenomenon was a distinct feature of strains $18 \mathrm{Z}$ and PG114 and was consistently observed in different experiments. It is not clear whether the rebound represents multiplication by surviving cocci or whether it is the result of yet another mechanism that permits enumeration of organisms previously inhibited by constituents initially present in the abscesses.

Unlike the other strains examined, $S$. aureus strain TG survived within abscesses for at least 5 weeks without a significant change in numbers. This strain produces the $\alpha, \beta$ and $\delta$ toxins as well as exfoliatin (epidermolytic toxin) and, unlike most other staphylococcal strains, is able to multiply in the subcutaneous tissue of neonatal mice (Kapral, 1976). Although subcutaneous multiplication appears to correlate with a strain's ability to produce exfoliatin, it is not known whether this toxin influences survival of staphylococci within peritoneal abscesses.

Staphylococci recovered from abscesses after 1-3 months were examined for haemolysin patterns in vitro. The patterns exhibited by the recovered cocci were identical to those of the organisms originally inoculated (see Kapral et al., 1980). The non-haemolytic variants $18 \mathrm{Z}-\mathrm{G}, 18 \mathrm{Z}-\mathrm{H}, \mathrm{P} 78-22$ and PG114-1 were destroyed more rapidly in abscesses than were the parent strains. To ascertain whether these mutants were inherently more susceptible to conditions within abscesses or were killed because they elicited a more vigorous host response, mixtures consisting of a haemolytic parent and its non-haemolytic variant were used to generate the abscesses. The results indicated that the survival of strains was essentially the same when present as mixtures or when present alone. The parent strain was unable to confer protection upon the simultaneously present non-haemolytic variant, and the variant was unable to potentiate killing of the parent. The preferential destruction of non-haemolytic variants suggested that these strains were inherently more sensitive to some host factor(s) within the abscess; it is possible that the previously detected cidal material is responsible for the effect.

Further studies revealed that sensitivity to the cidal system varied markedly among strains, but correlated with their ability to survive within abscesses. Those strains rapidly killed within abscesses were the most sensitive, whilst strains whose final destruction was delayed or strains that persisted were less sensitive. This correlation between in-vivo behaviour and in-vitro sensitivity supports the contention that the cidal material may contribute to the destruction of staphylococci in abscesses.

Originally, when bactericidal assays were conducted with saline as the diluent, some strains were found to be so resistant that an endpoint (LD50) could not be obtained. However, during the course of our studies we found that the cidal activity was increased in the presence of EDTA and high $\mathrm{NaCl}$ 
concentrations (Dye and Kapral, 1980). By performing assays in the presence of $1 \mathrm{M} \mathrm{NaCl}$ and 1mM EDTA it was possible to obtain a measurable endpoint for all the strains tested, thus enabling us to make more meaningful comparisons in sensitivity.

The cidal activity of abscess homogenates increased significantly after a period of incubation (activation). Although some cidal activity was always demonstrable in PAH before activation, a 24-hour period of activation caused a 3-20-fold increase in activity. The relationship of this activation process to events occurring naturally within abscesses is not yet understood, but recent findings suggest that the cidal substance (a lipid) is produced from an inactive precursor lipid. In the intact abscess, only about one-third of the precursor produced is ever converted to the cidal substance.

This investigation was supported by Public Health Service grant A1-7826 from The National Institute of Allergy and Infectious Disease.

\section{REFERENCES}

DYe, E. S. AND Kapral, F. A. 1980. Partial characterization of a bactericidal system in staphylococcal abscess. Infec. Immun., 30, 198.

FinNEY, D. J. 1971. Probit analysis, 3rd ed., Cambridge University Press, Cambridge.

KAPRAL, F. A. 1966. Clumping of Staphylococcus aureus in the peritoneal cavity of mice. $J$. Bact., 92, 1188.

KAPRAL, F. A. 1976. Subcutaneous multiplication of exfoliatin-producing staphylococci. Infec. Immun., 13, 682.

KAPRAL, F. A., Godwin, J. R. AND DYE, E. A. 1980. Formation of intraperitoneal abscesses by Staphylococcus aureus. Infec. Immun., 30, 204.

KAPRAL, F. A. AND Miller, M. M. 1971. Product of Staphylococcus aureus responsible for the scalded-skin syndrome. Infec. Immun., 4, 541.

KAPRAL, F. A. AND ShaYEGANI, M. 1959. Intracellular survival of staphylococci. J.exp. Med., $110,123$.

Melish, M. E. AND GlasGow, L. A. 1970. The staphylococcal scalded-skin syndrome. $N$. Engl. J. Med., 282, 1114. 